\title{
Coherent LiFi System with Spatial Multiplexing
}

\author{
Yinzhou Tan, Student Member, IEEE, and Harald Haas, Fellow, IEEE
}

\begin{abstract}
Coherent light fidelity (LiFi) systems which uses coherent detection at the receiver are attracting more and more interest as they provide many advantages over the conventional intensity modulation / direct detection (IM/DD) LiFi systems. Recent efforts to boost the performance of IM/DD LiFi systems include the usage of multiple-input-multiple-output (MIMO) technology, among which spatial multiplexing (SMX) has gained certain attention. This paper combines coherent detection and spatial multiplexing, and proposes a novel scheme called CD SMX (coherent-detection spatial-multiplexing) LiFi systems to improve $\mathrm{LiFi}$ performances. Compared with CD single-input-single-output (SISO) LiFi systems and CD spatial modulation (SM) LiFi systems, the proposed scheme provides a spectral efficiency gain. Unlike IM/DD or radio frequency (RF) SMX systems, the proposed CD SMX LiFi system is free from inter-channel interferences (ICI) due to the matching properties of coherent detection. The performance of the proposed CD SMX system is studied via both theoretical analysis and computer simulations. It is reported that the proposed system achieves a large gain over its IM/DD counterpart, and a moderate gain over a coherent system with SM.
\end{abstract}

Index Terms-optical communication, multiplexing, digital communication, MIMO systems

\section{INTRODUCTION}

Light fidelity (LiFi) was proposed as one solution to the exponentially increasing data traffic demand as the available radio frequency (RF) bandwidth has become insufficient [1]. In LiFi systems, data is conventionally modulated onto the intensity of the light emitting diode (LED) at the transmitter, and then demodulated using direct detection at the receiver. This principle is called intensity modulation / direct detection (IM/DD). The performance of IM/DD LiFi systems is greatly limited by the available bandwidth of the transmitting LEDs. Solutions to this issue include using alternative transmitters with a larger modulation bandwidth, such as laser diodes

Yinzhou Tan is with the LiFi Research and Development Centre (LRDC), the Institute for Digital Communication, the University of Edinburgh. E-mail: yinzhou.tan@ed.ac.uk; Harald Haas is with the Department of Electronic and Electrical Engineering, the University of Strathclyde. E-mail: harald.haas@strath.ac.uk
(LDs). The popular and commercially available phosphor-coated LEDs can provide a typical modulation bandwidth of around $20 \mathrm{MHz}$ with a blue filter, while LDs can provide modulation bandwidth in the $\mathrm{GHz}$ range while maintaining a relatively narrow wavelength profile [2]. In the meantime, the design of LEDs also faces an inherent tradeoff between modulation speed and output optical power level [2]. These circumstances have made laser based $\mathrm{LiFi}$ and visible light communication (VLC) systems more and more popular in recent years.

Instead of a laser-based IM/DD LiFi system, some researchers are now considering modulating the LD in both the amplitude and the phase of the laser field instead of simply modulating its intensity/power, and using coherent detection at the receiver. This results in a coherent-detection (CD) $\mathrm{LiFi}$ system [3]. In IM/DD systems, since the intensity is modulated, the signal must be real and positive; but in the CD system, it is the laser's electromagnetic (EM) field that is being modulated. Therefore, such restrictions no longer apply. Compared to direct detection, coherent detection provides better receiver selectivity and sensitivity and therefore better power efficiency [3]-[5], but does not directly increase the spectral efficiency of the system.

Besides using LDs as the light source or using coherent detection, a lot of effort has been made and many techniques have been applied to boost the spectral efficiency of LiFi systems, including multiple-input-multiple-output (MIMO) technology [1], [6], [7]. Various MIMO technologies have been studied. These include the repetition coding (RC) scheme where the same data is repeated by all transmitters, making the system robust to various transmitter-receiver alignments while requiring a large signal constellation size for high spectral efficiencies [7]. In contrast, spatial multiplexing (SMX) schemes send different data from every transmitter simultaneously, achieving multiplexing gains but causing performance-degrading inter chan- 
nel interferences (ICI) [7]. The spatial modulation (SM) scheme avoids ICI and increases both spectral and power efficiency, and has been attracting much research interest [8]-[11]. In SM, only one transmitter is activated at any time instance, and the transmitter indices are used to modulate data as well. The information rate is proportional to $\log _{2} N_{\mathrm{t}}$ in SM, while in SMX it scales with $\log _{2} \min \left(N_{\mathrm{t}}, N_{\mathrm{r}}\right)$ (where $N_{\mathrm{t}}$ and $N_{\mathrm{r}}$ are the number of the transmitters and of the receivers respectively). The spectral efficiency of SM systems increases with the number of transmitters at a cost of increased demodulation complexity [11]. The SMX scheme provides a spectral efficiency gain over the SM scheme, but its performance is greatly dependent on the channel condition.

In this work, we explore the possibility of combining coherent detection and MIMO techniques, to further boost the performance of a $\mathrm{LiFi}$ system. In a coherent optical system, the signal needs to be down-converted in the optical domain first by an optical local oscillator (LO) before its detection. This process is called photomixing. An essential requirement for the system to work is for the incident light field and the LO field to match in terms of both polarisation and spatial phase. That is to say, across the detecting plane, the phase difference between the two beams should be a constant and the EM fields of the two beams should have the same polarisation at any point. Imperfect matching would result in an attenuation to the amplitude of the desired data-carrying component of the generated photocurrent [4], [5], [12]. Imperfect matching, sometimes referred to as field mismatching, causes significant penalties to the system performance and sometimes makes it impossible to demodulate the data [4], [5]. The causes, effects, and the mitigation/compensation of field mismatching have been adequately addressed in fibre-optics and free-space optics (FSO) literature [4], [12], [13]. Since the perfect matching of the fields can be achieved by either adaptive methods as discussed in [13] or manual alignment, many researchers assume that there is perfect photomixing between the transmitter and the receiver as in [3], [5].

The effect of field mismatching has been revisited in this paper, and we introduced a correcting term to the existing formulae on photomixing efficiency in [4], [13], [14], to give a full account of the influences induced by field mismatching. We then proposed a novel type of $\mathrm{LiFi}$ system, the $\mathrm{CD}$ SMX (coherent-detection spatial multiplexing) LiFi system, where the matching properties are used to guarantee a diagonal channel matrix and an ICI-free MIMO system. The performance of the proposed system is theoretically analysed, and compared with IM/DD SM systems and CD SM systems via computer simulations.

Contributions: 1) We have revisited the photomixing issue of coherent optical systems, and have derived a refined formula for the photomixing efficiency to include the influences of the polarisation of the electromagnetic waves. By considering possibly different orientations of the electromagnetic waves, our refined formula gives a better account of the photomixing process than the existing formulae reported in [4], [13], [14]. This refinement, to the best of our knowledge, has never been reported before; 2) We proposed a new structure for $\mathrm{LiFi}$ systems, which combines the coherent detection and SMX schemes, namely the CD SMX LiFi system. Due to the photomixing properties of coherent optical signals, the proposed SMX system is free from ICI while still providing a spectral efficiency gain. The bit error ratio (BER) performance of this proposed system has been studied (using pairwise error probability (PEP) analysis and Monte Carlo simulations) to demonstrate that the proposed system provides better performance than coherent spatial modulation (SM) systems and IM/DD SM systems.

The rest of this paper is organised as follows. Section II revisits the principles of the coherent $\mathrm{LiFi}$ system and the effects of field mismatching. Section III describes the system structure of the CD SMX system. Then, section IV studies the performance of the proposed scheme and compares it with IM/DD SM systems and CD SM systems. The paper is concluded in section $\mathrm{V}$ along with an outline of our future works.

\section{PRELIMINARIES}

This section briefly describes how a LiFi system would work with the CD scheme and then revisits the field mismatching issue that has been discussed in literature such as [4] and [13].

\section{A. Principles of CD LiFi}

In CD LiFi systems, the data is modulated onto the EM fields of the laser instead of its intensity, 
therefore, it is necessary to consider the EM fields instead of simply considering the intensity. Denote the LD optical power as $P_{\mathrm{t}}$ and the channel direct current (DC) power gain as $G$ ( $G$ is the fraction of optical power detected by the photodiode (PD) when the signal light beam is vertically incident onto the detecting plane), then the total optical power of the signal across the detecting area is $P_{\mathrm{r}}=G P_{\mathrm{t}}$. Denote the total optical power of the LO across the detecting area as $P_{\mathrm{LO}}$. Assume the transmitter and the receiver are perfectly matched, so that both the received signal and the LO lasers are vertically incident onto the detecting plane, i.e. both fields have no spatial phase variation across the detecting area. Since the transmitter and the receiver are far enough away from each other, the incident signal light can be seen as a plane wave with uniformly distributed power across the detecting area. Assume the LO beam is collimated and close enough to the PD, then the LO light can also be seen as a plane wave with uniformly distributed power across the detecting area. Suppose the signal has been modulated by a constellation symbol $s=A e^{j \theta}$ where $j$ is the imaginary unit. Then, using the rootmean-square (rms) value for the field amplitudes and the phasor expression, the fields within the detecting area at a position $\boldsymbol{r}$, at time $t$, are given by the following equations:

- the electric field of the signal:

$$
\mathbf{E}_{\mathrm{s}}(\boldsymbol{r}, t)=\sqrt{\frac{Z G P_{\mathrm{t}}}{A_{\mathrm{d}}}} s e^{j \omega_{\mathrm{s}} t} \boldsymbol{e}
$$

- the magnetic field of the signal:

$$
\mathbf{H}_{\mathrm{s}}(\boldsymbol{r}, t)=\sqrt{\frac{G P_{\mathrm{t}}}{Z A_{\mathrm{d}}}} s e^{j \omega_{\mathrm{s}} t} \boldsymbol{h}
$$

- the electric field of the LO:

$$
\mathbf{E}_{\mathrm{LO}}(\boldsymbol{r}, t)=\sqrt{\frac{Z P_{\mathrm{LO}}}{A_{\mathrm{d}}}} e^{j \omega_{\mathrm{LO} t} \boldsymbol{e}}
$$

- and the magnetic field of the LO:

$$
\mathbf{H}_{\mathrm{LO}}(\boldsymbol{r}, t)=\sqrt{\frac{P_{\mathrm{LO}}}{Z A_{\mathrm{d}}}} e^{j \omega_{\mathrm{LO}} t} \boldsymbol{h}
$$

where $A_{\mathrm{d}}$ is the area of the detecting plane, $Z$ is the impedance, $\omega_{\mathrm{s}}$ and $\omega_{\mathrm{LO}}$ are the angular frequency of the signal and the LO respectively, and $\boldsymbol{e}$ and $\boldsymbol{h}$ are the unit vectors along the polarisation of the electric fields and the magnetic fields, respectively. Since both the signal and the LO lights are monochromatic EM waves, $\boldsymbol{e} \perp \boldsymbol{h}$. There is no phase difference between the signal and the LO, because we assume that the signal phase has been recovered by an optical phase lock loop (OPLL).

In the photomixing process, the electric fields add up linearly and so do the magnetic fields. So the time-averaged Poynting vector $\langle\mathbf{S}(\boldsymbol{r})\rangle$ of the total EM fields is given by [15]:

$$
\begin{aligned}
\langle\mathbf{S}(\boldsymbol{r})\rangle= & \left(\mathbf{E}_{\mathrm{s}}(\boldsymbol{r}, t)+\mathbf{E}_{\mathrm{LO}}(\boldsymbol{r}, t)\right) \\
& \times\left(\mathbf{H}_{\mathrm{s}}^{*}(\boldsymbol{r}, t)+\mathbf{H}_{\mathrm{LO}}^{*}(\boldsymbol{r}, t)\right)
\end{aligned}
$$

where '*' denotes complex conjugate, and ' $x$ ' denotes the cross product when two vectors are involved. Denote $\boldsymbol{s}=\boldsymbol{e} \times \boldsymbol{h}$, then $\boldsymbol{s}$ is the unit vector along the propagating direction of the 2 waves. Substituting the field expressions into (5), we get:

$$
\begin{aligned}
\langle\mathbf{S}(\boldsymbol{r})\rangle= & \frac{1}{A_{\mathrm{d}}}\left(P_{\mathrm{LO}}+G P_{\mathrm{t}} A^{2}\right. \\
& \left.+2 \sqrt{G P_{\mathrm{t}} P_{\mathrm{LO}}} A \cos \left(\omega_{\mathrm{IF}} t+\theta\right)\right) \boldsymbol{s}
\end{aligned}
$$

where $\omega_{\mathrm{IF}}=\omega_{\mathrm{s}}-\omega_{\mathrm{LO}}$ is the intermediate angular frequency (assume $\omega_{\mathrm{s}} \geqslant \omega_{\mathrm{LO}}$, and the case where $\omega_{\mathrm{s}}<\omega_{\text {LO }}$ can be similarly studied as cosine is an even function). For homodyne systems, $\omega_{\mathrm{IF}}=0$. The above result, (6), shows that the total field's optical power is also uniformly distributed across the detecting area because in the expression $\langle\mathbf{S}(\boldsymbol{r})\rangle$ does not depend on $\boldsymbol{r}$. As the light beams are also vertically incident, i.e., $\boldsymbol{s}=\boldsymbol{n}_{\mathrm{d}}$ where $\boldsymbol{n}_{\mathrm{d}}$ is the unit normal vector of the detecting plane (pointing inwards from the detection area), so the total received power is $A_{\mathrm{d}} \Re\{\langle\mathbf{S}\rangle\} \cdot \boldsymbol{n}_{\mathrm{d}}$ where $\Re\{\cdot\}$ is the real part of a complex variable. Therefore, the generated photocurrent is given by:

$$
\begin{aligned}
i_{\mathrm{p}}= & R P_{\mathrm{LO}}+R G P_{\mathrm{t}} A^{2} \\
& +2 R \sqrt{G P_{\mathrm{t}} P_{\mathrm{LO}}} A \cos \left(\omega_{\mathrm{IF}} t+\theta\right)+n
\end{aligned}
$$

where $R$ is the PD responsivity, and $n$ is the total noise term.

At the receiver side, the shot noise $n_{\mathrm{s}}$ will become dominant when the LO power is large enough, i.e. when $P_{\mathrm{LO}} \gg G P_{\mathrm{t}}$, and other noises (such as thermal noise and background noise) can be neglected [3], [5]. Therefore, for the coherent systems (both CD single-input-single-output (SISO) and CD SM) in the shot noise limited condition, $n \approx n_{\mathrm{s}}$. It has been 
demonstrated that the shot noise can be modelled by a white Gaussian process when a PD is used as the detector [5]. For the heterodyne system (passband system), the shot noise power is given by [3], [5]:

$$
\begin{aligned}
\sigma_{\mathrm{s}}^{2}=q B i_{\mathrm{p}}= & q B\left(R P_{\mathrm{LO}}+R G P_{\mathrm{t}} A^{2}\right. \\
& \left.+2 R \sqrt{G P_{\mathrm{t}} P_{\mathrm{LO}}} A \cos \left(\omega_{\mathrm{IF}} t+\theta\right)\right)
\end{aligned}
$$

where $q=1.60 \times 10^{-19} \mathrm{C}$ is the elementary charge and $B$ is the system bandwidth. For the homodyne system (baseband system), a multiplier of 2 should be applied to (8) but $B$ should be replaced by the baseband bandwidth, resulting in the same noise power as that of the heterodyne system. When $P_{\mathrm{LO}} \gg G P_{\mathrm{t}}$ as previously assumed, the term $R G P_{\mathrm{t}} A^{2}$ and the cosine term become negligible compared to $R P_{\mathrm{LO}}$. Therefore, the instantaneous photocurrent in (8) can be approximated by $R P_{\mathrm{LO}}$ when calculating the shot noise power and (8) now becomes [5]:

$$
\sigma_{\mathrm{s}}^{2} \approx q B R P_{\mathrm{LO}}
$$

When $P_{\mathrm{LO}} \gg G P_{\mathrm{t}}$ does not hold true, (8) should be used instead of (9). The component of interest of the photocurrent is the data-carrying alternating current (AC) term (the cosine term) in (7). This component is selected by a bandpass filter (BPF) from the photocurrent, and then an extra $-\pi / 2$ is added to the phase to create the quadrature component (for homodyne systems, this is done by using an extra LO with a $-\pi / 2$ phase shift and an extra PD, as the AC term would be reduced to a DC term). Then, they are used to demodulate the transmitted constellation point as in a RF heterodyne receiver. Assuming that the constellation has been normalised so that $\mathbb{E}\left[|s|^{2}\right]=\mathbb{E}\left[A^{2}\right]=1$ where $\mathbb{E}[\cdot]$ is the expectation, then from (7) and (9), the average signal-to-noise ratio (SNR) of the CD LiFi system (averaged over all possible constellation points) is given by:

$$
\mu=\frac{\left(2 R \sqrt{G P_{\mathrm{t}} P_{\mathrm{LO}}}\right)^{2} / 2}{q B R P_{\mathrm{LO}}}=\frac{2 R G P_{\mathrm{t}}}{q B}
$$

The SNR of the homodyne system should be $3 \mathrm{~dB}$ larger than $\mu$ given in (10), as the cosine term will be reduced to a DC term [5]. Without further indication, all CD systems in this study are heterodyne systems. Homodyne systems can be studied similarly.

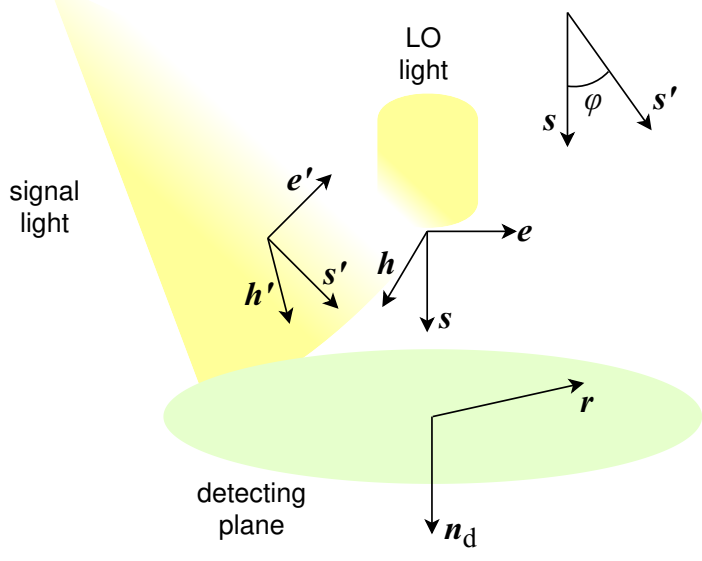

Fig. 1. Definition of the system vectors. All vectors except $\boldsymbol{r}$ shown in this figure have unit length. $\left(\boldsymbol{e}^{\prime}, \boldsymbol{h}^{\prime}, \boldsymbol{s}^{\prime}\right)$ describes the polarisation of the EM fields and the propagating direction of the signal, and $(\boldsymbol{e}, \boldsymbol{h}, \boldsymbol{s})$ describes those of the LO light. In our set up, $\boldsymbol{s}=\boldsymbol{n}_{\mathrm{d}} \cdot \varphi$ is the angle between $\boldsymbol{s}$ and $\boldsymbol{s}^{\prime}$

Combining (7) and (10), the sufficient statistic for the demodulator of the CD LiFi system is:

$$
y=\sqrt{\mu} s+z
$$

where $z \sim \mathcal{C N}(0,1)$

\section{B. Field mismatching}

In a coherent radio frequency (RF) system, the downconversion of the signal happens after the signal is received by a receiving antenna. In a coherent optical system, the downconversion happens simultaneously with the photomixing process, i.e. before signal detection. Therefore, as previously stated, the coherent optical system has stringent requirements that the spatial phase and the polarisation of the incident signal and those of the LO should match perfectly.

In the case where field mismatching exists, the equations describing the EM fields should be altered to account for the spatial phase variations caused. Since in practical applications the LO and the PD are fixed at the receiver, we assume that the LO light field is still vertically incident onto the detecting plane with uniformly distributed power $P_{\mathrm{LO}}$, i.e. (3) and (4) still stand. However, as the signal is now mismatched, (1) and (2) should be altered.

We assume that the PD is small enough compared to the transmission range, so that even when the 
signal is not vertically incident, the optical power of the signal is still approximately uniformly distributed across the detecting plane. As the propagating direction of the signal is now different, the righthand triplet $(\boldsymbol{e}, \boldsymbol{h}, \boldsymbol{s})$ should be replaced by another triplet $\left(\boldsymbol{e}^{\prime}, \boldsymbol{h}^{\prime}, \boldsymbol{s}^{\prime}\right)$. Denote the angle between $\boldsymbol{s}$ and $\boldsymbol{s}^{\prime}$ as $\varphi \in(0, \pi / 2]$. The relations of the mentioned vectors and the angle $\varphi$ are illustrated in Fig. 1. Then, taking $\boldsymbol{r}=\mathbf{0}$ as the reference point, the spatial phase variation $\exp \left(-j 2 \pi \boldsymbol{s}^{\prime} \cdot \boldsymbol{r} / \lambda_{\mathrm{s}}\right)$ where $\lambda_{\mathrm{s}}$ is the signal wavelength, should be introduced [13]. Hence, equations describing the signal fields are now:

- the electric field:

$$
\mathbf{E}_{\mathrm{s}}^{\prime}(\boldsymbol{r}, t)=\sqrt{\frac{Z G P_{\mathrm{t}}}{A_{\mathrm{d}}}} s e^{j \omega_{\mathrm{s}} t} e^{-j 2 \pi \boldsymbol{s}^{\prime} \cdot \boldsymbol{r} / \lambda_{\mathrm{s}}} \boldsymbol{e}^{\prime}
$$

- and the magnetic field:

$$
\mathbf{H}_{\mathrm{s}}^{\prime}(\boldsymbol{r}, t)=\sqrt{\frac{G P_{\mathrm{t}}}{Z A_{\mathrm{d}}}} s e^{j \omega_{\mathrm{s}} t} e^{-j 2 \pi \boldsymbol{s}^{\prime} \cdot \boldsymbol{r} / \lambda_{\mathrm{s}}} \boldsymbol{h}^{\prime}
$$

Using (5) to calculate the Poynting vector of the total EM fields, it now becomes:

$$
\begin{aligned}
\langle\mathbf{S}(\boldsymbol{r})\rangle= & \frac{G P_{\mathrm{t}} A^{2}}{A_{\mathrm{d}}} \boldsymbol{s}^{\prime}+\frac{P_{\mathrm{LO}}}{A_{\mathrm{d}}} \boldsymbol{s} \\
& +\frac{\sqrt{G P_{\mathrm{t}} P_{\mathrm{LO}}}}{A_{\mathrm{d}}} s e^{j \omega_{\mathrm{IF}} t} e^{-j 2 \pi s^{\prime} \cdot \boldsymbol{r} / \lambda_{\mathrm{s}}} \boldsymbol{e}^{\prime} \times \boldsymbol{h} \\
& +\frac{\sqrt{G P_{\mathrm{t}} P_{\mathrm{LO}}}}{A_{\mathrm{d}}} s^{*} e^{-j \omega_{\mathrm{IF}} t} e^{j 2 \pi s^{\prime} \cdot \boldsymbol{r} / \lambda_{\mathrm{s}}} \boldsymbol{e} \times \boldsymbol{h}^{\prime}
\end{aligned}
$$

As can be seen from (14), when the incident signal and the LO light are combined, the total optical power distribution across the detecting plane is no longer uniform, due to the spatial phase variation. Therefore, the total power received by the PD should now be calculated via integration across the detecting plane:

$$
\begin{aligned}
P_{\text {total }} & =\int_{A_{\mathrm{d}}} \Re\{\langle\mathbf{S}(\boldsymbol{r})\rangle\} \cdot \boldsymbol{n}_{\mathrm{d}} \mathrm{d} S \\
& =\Re\left\{\int_{A_{\mathrm{d}}}\langle\mathbf{S}(\boldsymbol{r})\rangle \cdot \boldsymbol{n}_{\mathrm{d}} \mathrm{d} S\right\}
\end{aligned}
$$

where '.' denotes the inner product of two vetcors when two vectors are involved.

Noticing $\boldsymbol{n}_{\mathrm{d}}=\boldsymbol{s}, \boldsymbol{s}^{\prime} \cdot \boldsymbol{s}=\cos \varphi$, and that both $(\boldsymbol{e}, \boldsymbol{h}, \boldsymbol{s})$ and $\left(\boldsymbol{e}^{\prime}, \boldsymbol{h}^{\prime}, \boldsymbol{s}^{\prime}\right)$ are right-hand triplet, we have $\left(\boldsymbol{e}^{\prime} \times \boldsymbol{h}\right) \cdot \boldsymbol{n}_{\mathrm{d}}=\boldsymbol{e}^{\prime} \cdot(\boldsymbol{h} \times \boldsymbol{s})=\boldsymbol{e}^{\prime} \cdot \boldsymbol{e}$ and $\left(\boldsymbol{e} \times \boldsymbol{h}^{\prime}\right) \cdot \boldsymbol{n}_{\mathrm{d}}=$ $\boldsymbol{h}^{\prime} \cdot(\boldsymbol{s} \times \boldsymbol{e})=\boldsymbol{h}^{\prime} \cdot \boldsymbol{h}$. Substituting these two relations and (14) into (15), we have:

$$
\begin{aligned}
& P_{\text {total }}=G P_{\mathrm{t}} A^{2} \cos \varphi+P_{\mathrm{LO}} \\
& +\frac{\sqrt{G P_{\mathrm{t}} P_{\mathrm{LO}}}}{A_{\mathrm{d}}}\left(\boldsymbol{e}^{\prime} \cdot \boldsymbol{e}\right) \Re\left\{s e^{j \omega_{\mathrm{IF}} t} \int_{A_{\mathrm{d}}} e^{-j 2 \pi \boldsymbol{s}^{\prime} \cdot \boldsymbol{r} / \lambda_{\mathrm{s}}} \mathrm{d} S\right\} \\
& +\frac{\sqrt{G P_{\mathrm{t}} P_{\mathrm{LO}}}}{A_{\mathrm{d}}}\left(\boldsymbol{h}^{\prime} \cdot \boldsymbol{h}\right) \Re\left\{s^{*} e^{-j \omega_{\mathrm{IF}} t} \int_{A_{\mathrm{d}}} e^{j 2 \pi \boldsymbol{s}^{\prime} \cdot \boldsymbol{r} / \lambda_{\mathrm{s}}} \mathrm{d} S\right\}
\end{aligned}
$$

The values of the integrals in (16) depend on the shape of the detecting area. In this study, we consider the simplest case, where the detecting area is circular with a diameter of $d_{\mathrm{d}}=2 \sqrt{A_{\mathrm{d}} / \pi}$. The centre of the circle is chosen as the reference point $\boldsymbol{r}=\mathbf{0}$. Then due to the symmetry, the two integrals will have the same real value given by:

$$
\int_{A_{\mathrm{d}}} e^{-j 2 \pi \boldsymbol{s}^{\prime} \cdot \boldsymbol{r} / \lambda_{\mathrm{s}}} \mathrm{d} S=A_{\mathrm{d}} \frac{2 J_{1}\left(\pi d_{\mathrm{d}} \sin \varphi / \lambda_{\mathrm{s}}\right)}{\pi d_{\mathrm{d}} \sin \varphi / \lambda_{\mathrm{s}}}
$$

where $J_{1}(x)$ is the first-order Bessel function of the first kind. Substituting (17) back into (16), the total received power by a circular detecting area is given by:

$$
\begin{aligned}
P_{\text {total }}= & G P_{\mathrm{t}} A^{2} \cos \varphi+P_{\mathrm{LO}} \\
& +2 \gamma_{0} \sqrt{G P_{\mathrm{t}} P_{\mathrm{LO}}} A \cos \left(\omega_{\mathrm{IF}} t+\theta\right)
\end{aligned}
$$

where $\gamma_{0}$ is the photomixing efficiency given by:

$$
\gamma_{0}=\frac{2 J_{1}\left(\pi d_{\mathrm{d}} \sin \varphi / \lambda_{\mathrm{s}}\right)}{\pi d_{\mathrm{d}} \sin \varphi / \lambda_{\mathrm{s}}} \frac{\left(\boldsymbol{e} \cdot \boldsymbol{e}^{\prime}+\boldsymbol{h} \cdot \boldsymbol{h}^{\prime}\right)}{2}
$$

It can be seen from (19) that $\left|\gamma_{0}\right| \leqslant 1^{1}$ and in the case of perfect matching $\left(\boldsymbol{e}=\boldsymbol{e}^{\prime}, \boldsymbol{h}=\boldsymbol{h}^{\prime}\right.$, and $\boldsymbol{s}=\boldsymbol{s}^{\prime}$ or equivalently $\varphi=0$ ), $\gamma_{0}=1$. When $\gamma_{0}<0$, it can be interpreted as an extra phase of $\pi$ since $\gamma_{0} \cos \left(\omega_{\mathrm{IF}} t+\theta\right)=-\gamma_{0} \cos \left(\omega_{\mathrm{IF}} t+\theta+\pi\right)$. Assume this phase is compensated for by the OPLL, we can replace $\gamma_{0}$ with its absolute value:

$$
\gamma=\left|\gamma_{0}\right|=\frac{2\left|J_{1}\left(\pi d_{\mathrm{d}} \sin \varphi / \lambda_{\mathrm{s}}\right)\right|}{\left|\pi d_{\mathrm{d}} \sin \varphi / \lambda_{\mathrm{s}}\right|} \frac{\left|\boldsymbol{e} \cdot \boldsymbol{e}^{\prime}+\boldsymbol{h} \cdot \boldsymbol{h}^{\prime}\right|}{2}
$$

where we do not cancel the factor 2 for better presentation, as both $\left(\left|\boldsymbol{e} \cdot \boldsymbol{e}^{\prime}+\boldsymbol{h} \cdot \boldsymbol{h}^{\prime}\right|\right) / 2$ and

\footnotetext{
${ }^{1}$ For a proof of this, please refer to Appendix A.
} 
$\left(2\left|J_{1}\left(\pi d_{\mathrm{d}} \sin \varphi / \lambda_{\mathrm{s}}\right)\right|\right) /\left(\left|\pi d_{\mathrm{d}} \sin \varphi / \lambda_{\mathrm{s}}\right|\right)$ take value between 0 and 1 . With the field mismatching present, the generated photocurrent now becomes:

$$
\begin{aligned}
i_{\mathrm{p}}^{\prime}= & R P_{\mathrm{LO}}+R G P_{\mathrm{t}} A^{2} \cos \varphi \\
& +2 \gamma R \sqrt{G P_{\mathrm{t}} P_{\mathrm{LO}}} A \cos \left(\omega_{\mathrm{IF}} t+\theta\right)+n
\end{aligned}
$$

Here we would like to point out, that unlike IM/DD LiFi systems where the receiver orientation is accounted for by the DC channel gain $G$, in our notation for the CD LiFi system it is accounted for by the photomixing efficiency $\gamma$, as non-vertically incident signals cause field mismatching. Again, considering the shot noise limited condition, the dominant component contributing to the shot noise is still $R P_{\mathrm{LO}}$ under previous assumptions, so the shot noise power remains the same as that given by (9). Compared to (7), it is easy to spot that the signal power has been reduced by a factor of $\gamma^{2}$. So the SNR in the case of field mismatching is given by:

$$
\mu^{\prime}=\gamma^{2} \mu=\gamma^{2} \frac{2 R G P_{\mathrm{t}}}{q B}
$$

and the sufficient statistic now becomes:

$$
y^{\prime}=\sqrt{\mu^{\prime}} s+z=\gamma \sqrt{\mu} s+z
$$

where still $z \sim \mathcal{C N}(0,1)$. If no signal is incident and only the LO is on, then the photocurrent reduces to $R P_{\mathrm{LO}}$ and both (11) and (23) reduce to $y=z$.

The influence of field mismatching is fully accounted for by the introduction of the photomixing efficiency, $\gamma$. Examining (20), we notice that ${ }^{2}$ :

$$
\left|\boldsymbol{e} \cdot \boldsymbol{e}^{\prime}+\boldsymbol{h} \cdot \boldsymbol{h}^{\prime}\right| \leqslant 1+\cos \varphi
$$

Hence, for a given $\varphi$ and all possible polarisation configurations, we have:

$$
\gamma \leqslant \frac{(1+\cos \varphi)}{2} \frac{2\left|J_{1}\left(\pi d_{\mathrm{d}} \sin \varphi / \lambda_{\mathrm{s}}\right)\right|}{\left|\pi d_{\mathrm{d}} \sin \varphi / \lambda_{\mathrm{s}}\right|}
$$

Recalling (5) and (15), it can be seen that the intermediate frequency (IF) component of the photocurrent can be equivalently calculated by:

$$
\begin{aligned}
i_{\mathrm{IF}}= & \frac{1}{Z} \Re\left\{\int_{A_{\mathrm{d}}} \mathbf{E}_{\mathrm{s}}^{\prime}(\boldsymbol{r}, t) \cdot \mathbf{E}_{\mathrm{LO}}^{*}(\boldsymbol{r}, t) \mathrm{d} S\right\} \\
& +Z \Re\left\{\int_{A_{\mathrm{d}}} \mathbf{H}_{\mathrm{s}}^{\prime}(\boldsymbol{r}, t) \cdot \mathbf{H}_{\mathrm{LO}}^{*}(\boldsymbol{r}, t) \mathrm{d} S\right\}
\end{aligned}
$$

\footnotetext{
${ }^{2}$ For a detailed analytical proof, please refer to Appendix B.
}

Since the amplitudes of the fields are proportional, some studies [4], [13], [14] are therefore defining the photomixing efficiency as the following expression (or its square put under the square root in (21)):

$$
\gamma^{\prime}=\frac{\left|\int_{A_{\mathrm{d}}} \mathbf{E}_{\mathrm{s}}^{\prime}(\boldsymbol{r}) \cdot \mathbf{E}_{\mathrm{LO}}^{*}(\boldsymbol{r}) \mathrm{d} S\right|}{\sqrt{\left(\int_{A_{\mathrm{d}}}\left|\mathbf{E}_{\mathrm{s}}^{\prime}(\boldsymbol{r})\right|^{2} \mathrm{~d} S\right)\left(\int_{A_{\mathrm{d}}}\left|\mathbf{E}_{\mathrm{LO}}^{*}(\boldsymbol{r})\right|^{2} \mathrm{~d} S\right)}}
$$

where $\mathbf{E}_{\mathrm{s}}^{\prime}(\boldsymbol{r})$ and $\mathbf{E}_{\mathrm{LO}}(\boldsymbol{r})$ are the spatial parts of $\mathbf{E}_{\mathrm{s}}^{\prime}(\boldsymbol{r}, t)$ and $\mathbf{E}_{\mathrm{LO}}(\boldsymbol{r}, t)$ respectively (omitting the $e^{j \omega t}$ term). This widely used definition of the photomixing efficiency, however, ignores the fact that $\boldsymbol{e}^{\prime} \cdot \boldsymbol{e}$ is not necessarily equal to $\boldsymbol{h}^{\prime} \cdot \boldsymbol{h}$ when field mismatching exists, i.e. the two terms in (26) are not generally equal ${ }^{3}$. The definition used in this study, $\gamma$, on the other hand, gives a complete account for the mismatching effect. As a matter of fact, the photomixing efficiency should be defined as:

$$
\frac{\left|\int_{A_{\mathrm{d}}} \mathbf{E}_{\mathrm{s}}^{\prime}(\boldsymbol{r}) \cdot \mathbf{E}_{\mathrm{LO}}^{*}(\boldsymbol{r}) \mathrm{d} S+Z^{2} \int_{A_{\mathrm{d}}} \mathbf{H}_{\mathrm{s}}^{\prime}(\boldsymbol{r}) \cdot \mathbf{H}_{\mathrm{LO}}^{*}(\boldsymbol{r}) \mathrm{d} S\right|}{2 \sqrt{\left(\int_{A_{\mathrm{d}}}\left|\mathbf{E}_{\mathrm{s}}^{\prime}(\boldsymbol{r})\right|^{2} \mathrm{~d} S\right)\left(\int_{A_{\mathrm{d}}}\left|\mathbf{E}_{\mathrm{LO}}^{*}(\boldsymbol{r})\right|^{2} \mathrm{~d} S\right)}}
$$

in general cases. The introduction of the correcting term in (28) has never been reported before, to the best of our knowledge. When the detecting area is circular with diameter $d_{\mathrm{d}}$ as previously assumed, (28) reduces to (20).

Now we examine the upper bound of $\gamma$ at a given $\varphi$ when the detecting area is circular, given by (25). Assume the diameter of the detecting area $d_{\mathrm{d}}$ is several centimetres, and the wavelength of the laser in the visible and the infrared spectra used in coherent LiFi systems are between several hundreds to approximately 1000 nanometres. Hence, the magnitude of $\pi d_{\mathrm{d}} / \lambda_{\mathrm{s}}$ is at least around 10000. This means that the $J_{1}(x) / x$ part would have multiple zeros within the interval $\varphi \in[0, \pi / 2)$. Given the angle between the propagation directions of the signal and the $\operatorname{LO} \varphi$, the upper bound of $\gamma$ can be easily calculated. An example for $d_{\mathrm{d}}=1 \mathrm{~cm}$ and $\lambda_{\mathrm{s}}=1000 \mathrm{~nm}$ is shown in Fig. 2. The calculation is done using Matlab.

It can be seen from Fig. 2 that although $\gamma$ fluctuates around 0 as $\varphi$ increased from 0 to $\pi / 2$ because

\footnotetext{
${ }^{3}$ For proof of this statement, please also refer to Appendix B.
} 


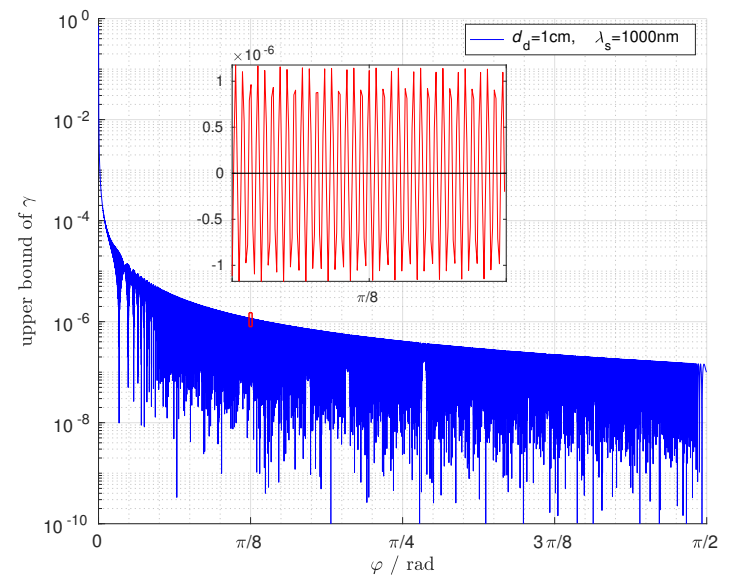

Fig. 2. An example of the upper bound of $\gamma$ given by (25) with different $\varphi$. The part in the red box $(0.3887<\varphi<0.3967)$ is zoomed in. In this zoomed-in box, (25) without the absolute value operation is plotted, instead of (25) itself, to show that the $J_{1}(x) / x$ component in (20) and (25) crosses zero multiple times. Notice that the $y$-axis in the figure is in logarithmic scale, while the $y$-axis in the zoomed-in box has linear scale. Distances between adjacent samples are $5 \times 10^{-5}$ rad in both the original figure and the zoomed-in box.

of the multiple zeros of $J_{1}(x) / x$, it drops drastically from 1 to around $10^{-6}$. The zoomed-in subplot in the figure shows the multiples zeroes of $\gamma$ in an exampling small interval $0.3887<\varphi<0.3967$, by plotting (25) without the absolute value operation instead of (25) itself (this difference is emphasised by using a different colour in the subplot). The mean value of the upper bound of $\gamma$ for $\varphi>0$ is $5.16 \times 10^{-5}$. The expected SNR penalty ${ }^{4}$ is approximately $47.33 \mathrm{~dB}$, if $\varphi$ is uniformly distributed in the interval $[0, \pi / 2)$. Reference [4] argues that a minimum photomixing efficiency (defined as the square of (27) in [4]) of $0.005 \%$ needs to be achieved for the coherent system in their set-up to have a receiver sensitivity gain over its direction detection counterpart [4]. This is approximately equivalent to a minimum efficiency $\gamma_{\min }=0.0071(42.97 \mathrm{~dB}$ SNR penalty) in our definition. With choices of $d_{\mathrm{d}}$ and $\lambda_{\mathrm{s}}$ as in Fig. 2, this requires approximately $\varphi<9 \times 10^{-4} \operatorname{rad}\left(0.0516^{\circ}\right)$. With this threshold being so strict and $\gamma$ being so small for $\varphi>0$, the $\mathrm{CD}$ system can be practically considered as nonfunctional when the fields are not perfectly matched. Therefore, from now on in this paper, we assume

\footnotetext{
${ }^{4}$ This is calculated by $10 \lg \left(\mathbb{E}\left[\widehat{\gamma}^{2}\right]\right)$ where $\widehat{\gamma}$ is the upper bound given by (25) and $\mathbb{E}[\cdot]$ is the expectation. The logarithm converts it to $\mathrm{dB}$ scale. The expectation is estimated by sample mean calculated in Matlab.
}

$\gamma \approx 0$ if the photomixing is imperfect.

\section{CD SMX SySTEM MODEL}

SMX systems are, in their nature, MIMO systems. Due to the matching requirement of the coherent optical systems, the matching pattern between the transmitters and the receivers needs to be specified before there can be any discussion regarding the system. As the LOs are fixed at the receiver side, it can be inferred that if one receiver perfectly matches more than one transmitter, this receiver must be equipped with more than one LOs with different orientations. This is because if there were more than one transmitter that perfectly matched one receiver with only one LO, then the said transmitters must have been deployed along a straight line. In this case, signals from farther transmitters would be blocked by nearer ones. If they were not along a straight line, then multiple beam altering components must have been used at the said receiver to match the signals to the fixed LO beam. Using more than one LO (and possibly more than one PD) or extra beam altering components at a single receiver would increase both the size and the complexity of the system, which may not be suitable for some practical LiFi applications. For this reason, in CD SMX systems, we apply the constraint that each receiver has only one LO and one PD, and is perfectly matched to only one transmitter; i.e. the matching pattern is one-on-one. Under this assumption, it follows that the CD SMX LiFi system is a $N \times N$ MIMO system where $N$ is the number of transmitters/receivers. In this study, we only consider $N \times N$ one-on-one-matched CD SMX LiFi systems.

Meanwhile, we are assuming that the CD SMX LiFi systems discussed in this study is based on infrared (IR) laser with wavelengths between 700 $\mathrm{nm}$ and $5000 \mathrm{~nm}$. The usage of the IR band in light communication (LC) has been included in the IEEE 802.11 Light Communications Amendment Task Group "bb", namely the IEEE 802.11bb [16]. It has also been agreed in IEEE $802.11 \mathrm{bb}$ that the $800 \mathrm{~nm}$ to $1000 \mathrm{~nm}$ band should be initially used in LC as silicon based detectors typically have peak responsivity in this band [17]. Using IR laser also avoids possible issues raised by laser illumination such as the speckle effect, which occurs when a fairly coherent light is either reflected from 
a rough surface or propagates through a medium with random refractive index [18]. Therefore, we consider the IR based LiFi system in this study.

\section{A. System model}

As previously stated, the CD SMX LiFi system is a $N \times N$ system. The SMX scheme is used, where the $N$ transmitters are simultaneously sending different data to the $N$ receivers. We label the transmitters and receivers with index $i \in\{1,2, \ldots, N\}$ and without loss of generality, assume that the $i$ th transmitter is perfectly matched to the $i$ th receiver, i.e. the photomixing efficiency between them is $\gamma_{i, i}=1$. Each transmitter has the optical power $P_{\mathrm{t}, i}$ and each receiver is equipped with one photodiode (PD) with detecting area $A_{\mathrm{d}, i}$, and one LO whose beam is vertically incident onto the detecting plane of the PD. The total LO power collected by the the PD is $P_{\mathrm{LO}, i}$ which is large enough. We make $P_{\mathrm{LO}, i}=P_{\mathrm{LO}}$ for all $i$, as when the LO power is large enough it only affects the shot noise power but not the SNR. This can be seen from (7) and (9): both the signal power and the shot noise power are proportional to $P_{\mathrm{LO}}$, so as long as $P_{\mathrm{LO}}$ is large enough, the SNR does not change with $P_{\mathrm{LO}}$. For all receivers, the $\mathrm{LO}$ is placed close to the $\mathrm{PD}$ and its beam is collimated, so that the PD of one receiver can not receive any light from the LO of another receiver. The light field of the LO and that of the signal are perfectly combined by a beam splitter without any losses.

For each pair of matched transmitters and receivers, we assume that they are located far enough from each other so that around the detecting plane the incident signal light can be approximated as a plane wave. For LDs with a Gaussian beam profile, this means that the transmission range should be much larger than the Rayleigh range of the source. For any pair of unmatched transmitters and receivers, we assume the photomixing efficiency between them is 0, following the discussion in Section I.B. In other words, the photomixing efficiency between the $i$ the receiver and the $j$ th transmitter is $\gamma_{i, j}=0$, if $i \neq j$.

At the transmitter side, we assume all transmitters use the same quadrature amplitude modulation (QAM) constellation of size $M$ (other forms of modulation can be discussed similarly). Neglecting the bandwidth limitations of the devices in the

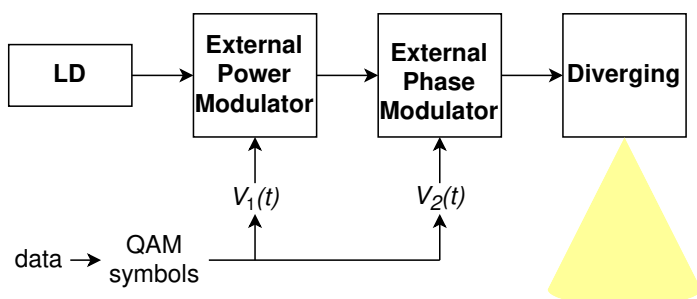

Fig. 3. Transmitting structure for a single transmitter.

system, the spectral efficiency is $\eta_{\mathrm{s}}=N \log _{2} M$ bits/s/Hz. The bit stream to be transmitted is divided into groups of length $\eta_{\mathrm{s}}$. Each group forms a timedomain symbol. In each symbol period, the $\eta_{\mathrm{s}}$ bits are further divided into $N$ sub-groups of length $\log _{2} M$ and each sub-group is mapped to a QAM symbol $s_{i}=A_{i} e^{j \theta_{i}}$. The $i$ th QAM symbol is to be transmitted by the $i$ th transmitter. The transmitters are modulated by voltage-driven external modulators [3], [5]. Two voltage signals, $V_{1}(t)$ and $V_{2}(t)$, are generated, corresponding to the power and the phase of the QAM symbols respectively. $V_{1}(t)$ and $V_{2}(t)$ are then used to drive the external power modulator and the external phase modulator respectively. The modulated signal is then diverged to provide a coverage. The transmitting structure is shown in Fig. 3.

The channel is modelled as a flat single-tap channel, as we neglect multipath effects. The receiver then decides, in each symbol period, which constellation points are transmitted by the $N$ transmitters, to reconstruct the original bit stream. A brief diagram showing the overall system structure is given in Fig. 4.

In our previous discussions, the transmitted signals are represented by their field strength. The relation between the field strength and the photocurrent is non-linear as seen in (7) and (21). In order to obtain a linear relation, we do not consider the actual wave form (field strength) of the signals in our system model. At the transmitter side, we consider the transmitted constellation symbols $s_{i}$; at the receiver side, we consider the sufficient statistic given by (11) or (23). Denote the transmitted 'signal' with a $N \times 1$ vector $\mathbf{x}=\left[s_{1}, s_{2}, \ldots, s_{N}\right]^{\mathrm{T}}$. The sufficient statistics at the receiver side can be expressed by a $N \times 1$ vector $\mathbf{y}$. Based on previous discussions, we have:

$$
\mathbf{y}=\mathbf{H x}+\mathbf{n}
$$




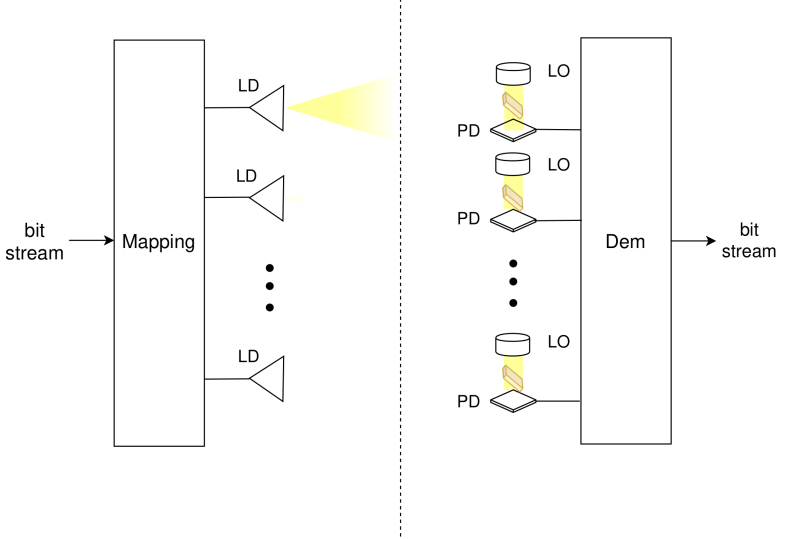

Fig. 4. The CD SMX LiFi system structure. The transmitter side is on the left of the dotted line and the receiver side is on the right. At the receiver side, between the PD and the LO, is the power splitter to combine the incident signal and the LO light. 'Dem' stands for demodulator.

where $\mathbf{H}$ is the $N \times N$ equivalent channel matrix whose elements are given by:

$$
h_{i, j}=\gamma_{i, j} \sqrt{\mu_{i, j}}
$$

where $\gamma_{i, j}$ is the photomixing efficiency between the $i$ th receiver and the $j$ th transmitter, $\mu_{i, j}$ is the SNR (averaged over all possible symbols in the constellation) between the $i$ th receiver and the $j$ th transmitter assuming perfect photomixing, for all $i, j \in\{1,2, \ldots, N\}$, and $\mathbf{n}$ is the noise vector, whose elements are independent identically distributed (i.i.d.) complex standard normal random variables $\left(\mathrm{n}_{i} \sim \mathcal{C N}(0,1)\right.$ where $\mathrm{n}_{i}$ is the $i$ th element of n). Recalling (22), it is easy to see:

$$
\mu_{i, j}=\frac{2 R_{i} G_{i, j} P_{\mathrm{t}, j}}{q B}
$$

where $R_{i}$ is the responsivity of the $i$ th receiver and $G_{i, j}$ is the DC optical power gain between the $i$ th receiver and the $j$ th transmitter. Recalling our previous discussion on $\gamma_{i, j}$, the conclusion regarding mismatching can be easily extended to the case where multiple signals mix together with one LO, by considering multiple fields instead of 2 in (5). Similarly, only the matched terms will remain nonzero. Therefore,

$$
h_{i, j}=\delta_{i j} \sqrt{\mu_{i, j}}
$$

where $\delta_{i j}$ is the Kronecker delta ( 1 for $i=j$ and 0 otherwise). From now on, we denote $\mu_{i, i}=\mu_{i}$ for convenience. This means that the equivalent

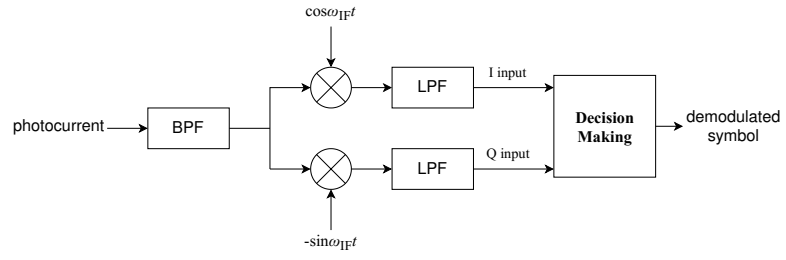

Fig. 5. Receiving structure for heterodyne receiving.

channel matrix $\mathbf{H}$ is diagonal. The results in (16) and (21) can be easily extended to cases where multiple laser beams are mixing, where the number of DC terms is equal to that of the laser beams and only the cosine terms corresponding to the photomixing between two matched laser beams will be non-zero. Under the previous assumption that the LO power is large enough, the DC terms corresponding to the signal lasers become negligible compared to the one corresponding to the LO. Hence, even when multiple transmitters are operating, our previously derived equations on the SNR still stand. With the one-on-one matching pattern and the channel matrix $\mathbf{H}$ being diagonal, the proposed CD SMX system is free from inter-channel interferences (ICI) ${ }^{5}$.

To calculate the sufficient statistics for demodulation, a structure similar to a heterodyne receiver is needed for each receiver. The photocurrent is first filtered by a BPF to extract the IF component, and then again down-converted by an electrical LO of angular frequency $\omega_{\mathrm{IF}}$ and filtered by a low pass filter (LPF). The quadrature component is acquired by adding a $\pi / 2$ phase to the electrical LO, and then the electrical down-conversion and LPF filtering is carried out. The two LPFs acquire the I input and the $\mathrm{Q}$ input respectively which are used for decision making to determine the transmitted symbol. A brief diagram of this structure is given in Fig. 5.

In the CD SMX system, the matching pattern is known to both the transmitter and the receiver side. As the system is free from ICI, the $N$ receivers can demodulate the signals individually and then combine the bits according to the indices of their matched transmitters. The CD SMX system uses a maximum a posteriori (MAP) estimator at each receiver to demodulate the QAM symbol from the sufficient statistic. To use the vector form as in (29):

$$
\widehat{\mathbf{x}}=\arg \max _{\mathbf{x} \in \mathcal{X}} p_{\mathbf{y}}(\mathbf{y} \mid \mathbf{x}) p_{\mathbf{x}}(\mathbf{x})
$$

\footnotetext{
${ }^{5}$ For a detailed explanation, please refer to Appendix C
} 
where $\widehat{\mathbf{x}}$ is the demodulation result, $\mathcal{X}$ is the set of all possible $\mathbf{x}, p_{\mathbf{y}}(\mathbf{y} \mid \mathbf{x})$ is the probability density function (pdf) of $\mathbf{y}$ conditioned on $\mathbf{x}$, and $p_{\mathbf{x}}(\mathbf{x})$ is the pdf of $\mathbf{x}$. In IM/DD systems which use DC-biased optical orthogonal frequency division multiplexing (DCO-OFDM), the temporal symbols follow a Gaussian distribution [3], [19]. In CD systems, the above MAP problem is equivalent however, to solving the maximum likelihood (ML) problem since all possible QAM symbols have the same probability of being chosen $\left(p_{\mathbf{x}}(\mathbf{x})=1 / M\right)$ :

$$
\widehat{\mathbf{x}}=\arg \max _{\mathbf{x} \in \mathcal{X}} p_{\mathbf{y}}(\mathbf{y} \mid \mathbf{x})=\arg \min _{\mathbf{x} \in \mathcal{X}}\|\mathbf{y}-\mathbf{H x}\|^{2}
$$

\section{PERFormances of CD SMX LiFi}

\section{A. Theoretical analysis}

Following (23), the pdf of $\mathbf{y}$ conditioned on $\mathbf{x}$ is given by

$$
p_{\mathbf{y}}(\mathbf{y} \mid \mathbf{x})=\frac{1}{(2 \pi)^{N}} \exp \left(-\frac{1}{2}\|\mathbf{y}-\mathbf{H x}\|^{2}\right)
$$

As the system is ICI-free, the demodulation at each receiver can be considered independent. Each receiver demodulates a QAM symbol within one symbol period, so the system's bit error ratio (BER) is equivalent to the average of the BERs at all receivers. We consider the $i$ th receiver as an example, and calculate the pair-wise error probability (PEP) to obtain its BER.

From previous discussions, $y_{i} \sim \mathcal{C N}\left(\sqrt{\mu_{i}} s_{i}, 1\right)$ where $y_{i}$ is the sufficient statistic at the $i$ th receiver, $s_{i}$ is the transmitted QAM symbol from the $i$ th transmitter in this symbol period. The PEP of mistaking $s_{j}$ for $s_{i}(j \neq i)$ is given by $\operatorname{Pr}\left(\widehat{s}_{i}=s_{j} \mid s_{i}\right)=$ $\operatorname{Pr}\left(s_{j}=\arg \min _{\mathcal{S}}\left|y-\sqrt{\mu_{i}} s\right|^{2}\right)$ where $\mathcal{S}$ is the set of all QAM symbols.

We first calculate $d_{s_{i}}\left(s_{1}, s_{2}\right)=\left|y_{i}-\sqrt{\mu_{i}} s_{1}\right|^{2}-$ $\left|y_{i}-\sqrt{\mu_{i}} s_{2}\right|^{2}$ where $y_{i}$ is conditioned on $s_{i}$. In the notation of $d_{s_{i}}\left(s_{1}, s_{2}\right)$ and in following equations, the $s_{i}$ in the suffix means 'conditioned on $s_{i}$ being transmitted'. It is easy to see that

$$
\begin{aligned}
& d_{s_{i}}\left(s_{1}, s_{2}\right)= \\
& \mu_{i}\left(\left|s_{1}\right|^{2}-\left|s_{2}\right|^{2}\right)-2 \sqrt{\mu_{i}} \Re\left\{y\left(s_{1}-s_{2}\right)^{*}\right\}
\end{aligned}
$$

As $s_{1}$ and $s_{2}$ are determinant and $y_{i}$ is complex Gaussian, $d_{s_{i}}\left(s_{1}, s_{2}\right)$ is also Gaussian with mean:

$$
\begin{aligned}
& u_{s_{i}}\left(s_{1}, s_{2}\right)= \\
& \mu_{i}\left(\left|s_{1}\right|^{2}-\left|s_{2}\right|^{2}\right)-2 \mu_{i} \Re\left\{s_{i}\left(s_{1}-s_{2}\right)^{*}\right\}
\end{aligned}
$$

and variance:

$$
\sigma_{s_{i}}^{2}\left(s_{1}, s_{2}\right)=2 \mu_{i}\left|s_{1}-s_{2}\right|^{2}
$$

Combining the above results, it can be seen that conditioned on $s_{i}$ :

$$
\begin{aligned}
& \operatorname{Pr}\left(\left|y_{i}-\sqrt{\mu_{i}} s_{1}\right|^{2}>\left|y_{i}-\sqrt{\mu_{i}} s_{2}\right|^{2}\right) \\
& =\operatorname{Pr}\left(d_{s_{i}}\left(s_{1}, s_{2}\right)>0\right) \\
& =Q\left(-\frac{u_{s_{i}}\left(s_{1}, s_{2}\right)}{\sigma_{s_{i}}\left(s_{1}, s_{2}\right)}\right)
\end{aligned}
$$

where $Q(x)$ is the Q-function. When the $\mathrm{SNR}$ is reasonably high, we would have $\operatorname{Pr}\left(\hat{s}_{i}=s_{j} \mid s i\right)=$ $\operatorname{Pr}\left(d_{s_{i}}\left(s 1, s_{j}\right)>0 \wedge \ldots \wedge d_{s_{i}}\left(s_{j-1}, s_{j}\right)>0 \wedge\right.$ $\left.d_{s_{i}}\left(s_{j+1}, s_{j}\right)>0 \wedge \ldots \wedge d_{s_{i}}\left(s_{M}, s_{j}\right)>0\right) \approx$ $\prod_{1 \leqslant k \leqslant M, k \neq j} \operatorname{Pr}\left(d_{s_{i}}\left(s_{k}, s_{j}\right)>0\right)$. Therefore, in high SNR scenario, the PEP $\operatorname{Pr}\left(\widehat{s}_{i}=s_{j} \mid s_{i}\right)$ can now be estimated by:

$$
\operatorname{Pr}\left(\widehat{s}_{i}=s_{j} \mid s_{i}\right) \approx \prod_{1 \leqslant k \leqslant M, k \neq j} Q\left(-\frac{u_{s_{i}}\left(s_{k}, s_{j}\right)}{\sigma_{s_{i}}\left(s_{k}, s_{j}\right)}\right)
$$

The error probability conditioned on $s_{i}$ can be directly estimated via:

$$
\begin{aligned}
& \operatorname{Pr}\left(\widehat{s}_{i} \neq s_{i} \mid s_{i}\right) \approx 1-\prod_{1 \leqslant j \leqslant M, j \neq i} \operatorname{Pr}\left(d_{s_{i}}\left(s_{j}, s_{i}\right)>0\right) \\
& =1-\prod_{1 \leqslant j \leqslant M, j \neq i} Q\left(-\frac{u_{s_{i}}\left(s_{j}, s_{i}\right)}{\sigma_{s_{i}}\left(s_{j}, s_{i}\right)}\right) \\
& =1-\prod_{1 \leqslant j \leqslant M, j \neq i} Q\left(-\sqrt{\frac{\mu_{i}}{2}}\left|s_{j}-s_{i}\right|\right)
\end{aligned}
$$

The BER of the system can be calculated based on the PEP given by (40). Denote the Hamming distance between the QAM symbols $s_{1}$ and $s_{2}$ as $h\left(s_{1}, s_{2}\right)$, then when $s_{i}$ is mistaken for $s_{j}$, the error ratio is:

$$
e\left(s_{i}, s_{j}\right)=\frac{h\left(s_{i}, s_{j}\right)}{\log _{2} M}
$$

Averaging over all possible errors and all possible transmitted QAM symbols, the BER conditioned at the $i$ th receiver is: 
$\operatorname{BER}^{(i)}=\frac{1}{M} \sum_{s_{i} \in \mathcal{S}} \sum_{s_{j} \in \mathcal{S}, s_{j} \neq s_{i}} \frac{h\left(s_{i}, s_{j}\right)}{\log _{2} M} \operatorname{Pr}\left(\widehat{s}_{i}=s_{j} \mid s_{i}\right)$

It can be seen that the term $\mathrm{BER}^{(i)}$ is dependent on $\mu_{i}$ and the bit labelling rule of the system. Averaging over all receivers, the system's BER is:

$$
\mathrm{BER}=\frac{1}{N} \sum_{i=1}^{N} \mathrm{BER}^{(i)}
$$

In the simplest case where all transmitters use the same bit labelling rule and all $\mu_{i}$ are equal, it is easy to see that $\mathrm{BER}=\mathrm{BER}^{(1)}=\mathrm{BER}^{(2)}=\ldots=$ $\operatorname{BER}^{(N)}$.

\section{B. Simulations}

Using (44) to estimate the system BER involves the calculation of $M(M-1)$ PEPs, which requires even more calculations for $u_{s}\left(s_{1}, s_{2}\right)$ and $\sigma_{s}^{2}\left(s_{1}, s_{2}\right)$ from (37) and (38). This requires a lot of computations especially when $M$ is large. In this study, apart from calculating from (44), we also examine the system's performance using numerical simulations.

The numerical estimation of BER is evaluated by Matlab simulations, using the Monte Carlo method. A random bit stream is generated, and then grouped to the transmitters and mapped to the QAM symbols. The symbols are then used to modulate the optical carrier, and the modulated signal is transmitted, received, detected and demodulated to reconstruct the bit stream. The reconstructed bit stream is compared with the original bit stream to obtain an estimation of the BER. The above procedure is repeated many times while averaging the results, to provide a more accurate estimation of the system's BER.

In our simulations, we are considering a simple SMX scheme without any pre-coding or equalisation techniques. For simplicity of calculation, we consider the simplest case, where all transmitters have the same transmitting power $P_{\mathrm{t}}$, all receivers have the same detecting area $A_{\mathrm{d}}$ and responsivity, and for any $1 \leqslant i, j \leqslant N, G_{i, j}=G$. Therefore, we would have $\mu_{1}=\mu_{2}=\ldots=\mu_{N}=\mu$. We are also considering the shot noise limited condition (i.e. neglecting other noises such as the background noise and considering only the shot noise and the thermal noise), as in high SNR scenarios the shot noise becomes dominant. The shot noise power is calculated by (8), and the thermal noise power for the coherent system is calculated by [20]:

$$
\sigma_{\mathrm{t}}^{2}=\frac{2 k_{\mathrm{B}} B T}{R_{\mathrm{L}}}
$$

where $k_{\mathrm{B}}=1.38 \times 10^{-23} \mathrm{~J} / \mathrm{K}$ is the Boltzmann constant, $T$ is the temperature in $\mathrm{K}$ and $R_{\mathrm{L}}$ is the load resistance. Please note [20, eq. (4)] is written for the baseband IM/DD system while (45) is written for the coherent system. The parameters used in our simulations are listed in Table. I.

TABLE I

SIMULATION PARAMETERS

\begin{tabular}{c|c|c}
\hline Parameter & Notation & Value \\
\hline PD responsivity & $R$ & $0.28 \mathrm{~A} / \mathrm{W} \mathrm{[20]}$ \\
\hline Bandwidth & $B$ & $40 \mathrm{MHz}[20]$ \\
\hline DC channel gain & $G$ & $-60 \mathrm{~dB} \mathrm{[3]}$ \\
\hline LO power (only for CD systems) & $P_{\mathrm{LO}}$ & $10 \mathrm{dBm}$ \\
\hline Temperature & $T$ & $295 \mathrm{~K} \mathrm{[20]}$ \\
\hline Load resistance & $R_{\mathrm{L}}$ & $10 \mathrm{k} \Omega[20]$ \\
\hline Transmitter/receiver number & $N$ & 4 \\
\hline
\end{tabular}

It is necessary to point out, that the $40 \mathrm{MHz}$ bandwidth in Table. $I$ is the passband bandwidth of the CD SMX system. The $20 \mathrm{MHz}$ bandwidth used in [20] is the baseband bandwidth of the IM/DD system. In our study, by setting the passband bandwidth to $40 \mathrm{MHz}$ and the baseband bandwidth to $20 \mathrm{MHz}$, we guarantee the same bandwidth consumption for all compared systems.

We compare the proposed CD SMX system with a CD SM (spatial modulation) system and an IM/DD SM system, with the same number of transmitters/receivers, DC channel gains, bandwidth consumption and bit rate. As the IM/DD SMX system usually suffers from ICI and its performance is greatly dependent on channel conditions, we are considering an IM/DD SM system instead. The IM/DD SM system uses the DCO-OFDM scheme with 1024 frequency domain subcarriers and the same QAM constellation on each subcarrier. The CD SM system uses the same system model as the CD SMX system, except that it uses the SM scheme where only one transmitter is active at any time. The respective spectral efficiency in bits per symbol of the 3 systems with $N$ transmitters/receivers and QAM constellation size $M$ are given by [19]: $\eta_{\text {CD-SMX }}=N \log _{2} M, \eta_{\text {CD-SM }}=\log _{2} N+\log _{2} M$, and $\eta_{\text {IMDD-SM }}=\log _{2} N+\frac{1}{2} \log _{2} M$. 


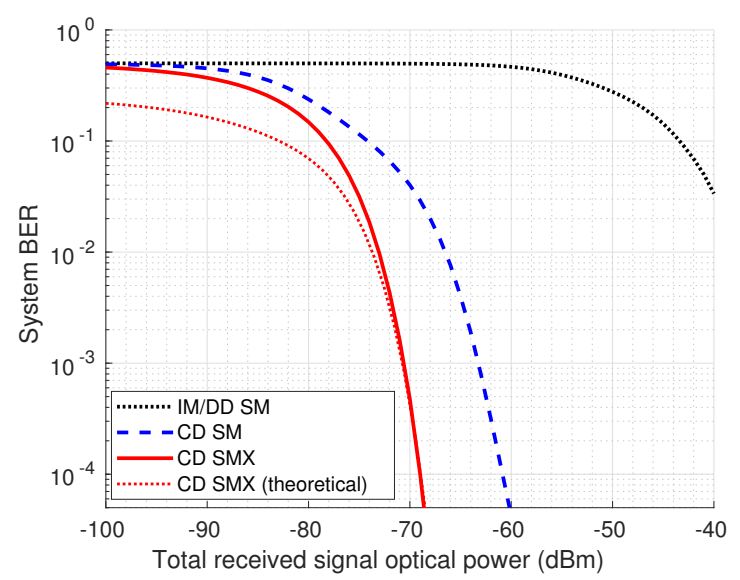

Fig. 6. BER comparison of CD SMX with CD SM and IM/DD SM at the same spectral efficiency, plotted against total received signl optical power.

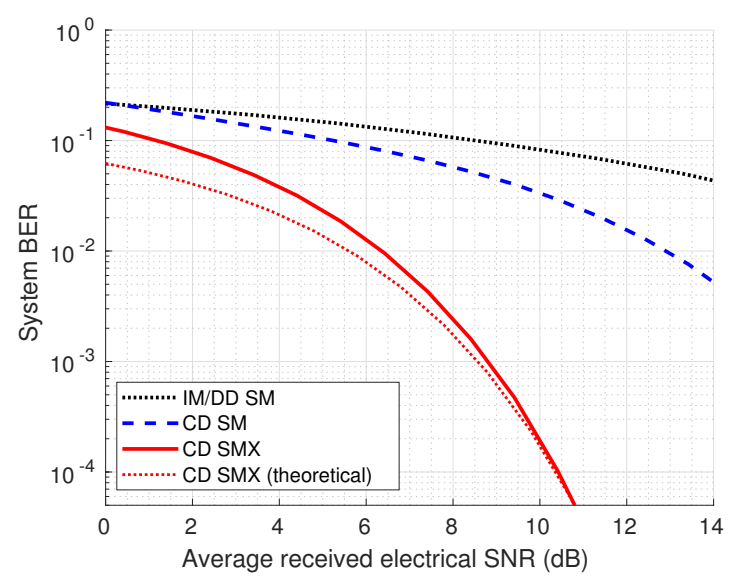

Fig. 7. BER comparison of CD SMX with CD SM and IM/DD $\mathrm{SM}$ at the same spectral efficiency, plotted against received electrical SNR (for the SMX system, the SNR is the SNR at one receiver).

Therefore, in our simulation, in order to guarantee the same bit rate at the same bandwidth consumption, we choose $N=4$ for all systems, and $M=4$ for CD SMX, $M=64$ for CD SM, and $M=4096$ for IM/DD SM with DCO-OFDM, resulting in a spectral efficiency of 8 bits per symbol. We also ensure that all three systems have the same total transmitting optical power, which means that each transmitter of the CD SMX system is operating at an optical power $P_{\mathrm{t}} / N$ if the CD SM system and the IM/DD SM system are transmitting at power $P_{\mathrm{t}}$. The BER results are shown in Fig. 6.

In Fig. 6, the BER is plotted against the total received signal power; in Fig. 7, the BER is plotted against the received electrical SNR (for the SMX system, the SNR is defined by the average

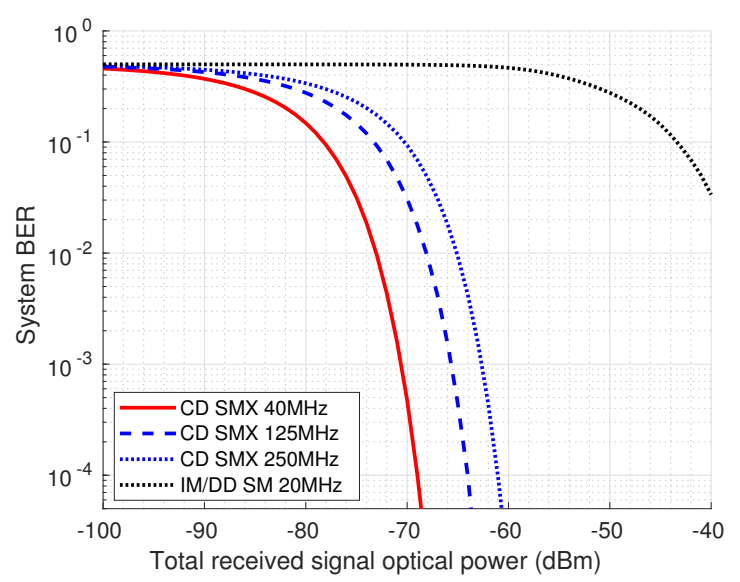

Fig. 8. BER comparison between CD SMX systems with different bandwidth. As the benchmark IM/DD SM system is a baseband system, the $20 \mathrm{MHz}$ IM/DD SM system actually consume the same bandwidth as the $40 \mathrm{MHz} \mathrm{CD}$ system.

SNR of each receiver. In our simulation set-up, all receivers achieve the same $\mathrm{SNR}$ ). It can be seen that the proposed CD SMX system has superior performance to both the CD SM system and the IM/DD SM system. The red dotted line is the theoretical results calculated with (40), (43) and (44). As we pointed out when deriving (40), this estimation is only valid for high SNR scenarios. The theoretical results match closely with the numerical results in high SNR scenarios. Compared to the IM/DD system, the proposed system has a power gain of over $30 \mathrm{~dB}$ and a SNR gain of about $10 \mathrm{~dB}$. It can be seen that despite the receiver sensitivity gain of coherent detection, the reported large power gain is mainly due to the huge spectral efficiency gain of the SMX scheme. With a much smaller QAM constellation, the SMX scheme can achieve a satisfactory BER performance at a lower SNR and received power. Compared to the CD SM system, the proposed system achieves a gain of approximately $7 \mathrm{~dB}$ around a BER of $10^{-3}$. This is also due to the spectral efficiency gain by using the SMX scheme. Our proposed novel system is able to exploit the full potential of the SMX technology, as the system is ICI-free because of the matching properties of coherent optical systems.

The LDs used for the CD systems have a wider bandwidth than LEDs. We also compare CD SMX systems with different bandwidths (bit rates) while keeping other parameters and conditions the same, as presented in Table. I. We include the results for the IM/DD SM system as a benchmark, as they 
usually have different bandwidths. The results are shown in Fig. 8.

From Fig. 8, it can be seen that the proposed CD SMX system can achieve a better BER performance than IM/DD SM systems with a higher bandwidth. Among CD SMX systems, the conclusion can be drawn that the smaller the bandwidth, the better the BER performance. This is easily understood, as a larger bandwidth results in a larger noise power. Comparing the $20 \mathrm{MHz}$ IM/DD SM system with the $250 \mathrm{MHz}$ CD SMX system, we can see that a gain of over $20 \mathrm{~dB}$ is achieved. This means that the proposed CD SMX system is able to provide a higher data rate with a smaller bandwidth, and therefore becomes desirable for next generation high speed communications.

\section{CONCLUSION AND FUtURE WORKS}

In this study, we firstly addressed the field mismatching in coherent detection. We gave a full account of the influence of polarisation and phase mismatching by introducing a correcting term into the existing formulae, which has never been provided before to the best of our knowledge. We then proposed a novel structure, the CD SMX scheme which combines the SMX and the coherent detection technologies, of LiFi systems. Due to the matching requirement between the signal and the LO in coherent detection, the proposed CD SMX system can easily achieve a diagonal channel matrix by fixing the LOs and restricting the number of LOs at each receiver to one. In this case, the system is free from ICI. The system provides both a spectral efficiency gain by SMX and a receiver sensitivity gain by coherent detection.

The BER performance of the proposed system is evaluated via both theoretical analysis and computer simulations. Numerical results for the simple scenario where each receiver receives the same optical power, are obtained via Matlab using the Monte Carlo method. Compared to the CD SM system, the proposed system achieves a large spectral efficiency gain which results in a gain of around 7 dB. Compared to the DCO-OFDM based IM/DD SM system, our proposed system achieves a considerably large power gain of over $30 \mathrm{~dB}$ and a SNR gain of about $10 \mathrm{~dB}$, due to the significant gain of receiver sensitivity by the coherent receiver and the spectral efficiency gain by SMX over SM.
However, the total power consumption of the CD SMX system increases linearly with the number of transmitters/receivers. In the comparison of systems with different bandwidth, it is demonstrated that the proposed CD SMX LiFi system is able to outperform its IM/DD SM counterpart even when the CD SMX LiFi system has larger bandwidth (bit rate). This makes the proposed system desirable for next generation high speed communications.

This is our first work on CD SMX systems. In the future, we will study the system under various channel conditions. We will also study the possibility of adapting variations of MIMO techniques that have been used in IM/DD systems into coherent $\mathrm{LiFi}$ systems, and their respective performances.

\section{APPENDIX A}

We now prove $\left|\gamma_{0}\right| \leqslant 1$ given by (19). As the vectors in (19) are all unit vectors (as defined), it can be seen that $\left(\boldsymbol{e} \cdot \boldsymbol{e}^{\prime}+\boldsymbol{h} \cdot \boldsymbol{h}^{\prime}\right) / 2 \leqslant(1+1) / 2=1$.

The first term involves the first-order Bessel function of the first kind, and the minimum of it is equivalent to that of $2 J_{1}(x) / x$ for any real number $x$. For $x=0$, it is defined as:

$$
\left.\frac{2 J_{1}(x)}{x}\right|_{x=0} \triangleq \lim _{x \rightarrow 0} \frac{2 J_{1}(x)}{x}=1
$$

For integer $n, J_{n}(x)$ has the following recurrence relations:

$$
\begin{aligned}
& \frac{2 n J_{n}(x)}{x}=J_{n-1}(x)+J_{n+1}(x) \\
& \frac{\mathrm{d}}{\mathrm{d} x}\left[x^{-n} J_{n}(x)\right]=x^{-n} J_{n+1}(x)
\end{aligned}
$$

So, letting $n=1$, we have $2 J_{1}(x) / x=$ $J_{0}(x)+J_{2}(x)$ and $\left(2 J_{1}(x) / x\right)^{\prime}=2 J_{2}(x) / x$ where ' $\left(2 J_{1}(x) / x\right)^{\prime}$ ' is the derivative of $2 J_{1}(x) / x$.

As $2 J_{1}(x) / x$ is even, we only need to consider $x \geqslant 0$. Let its derivative be 0 , we know that the local extrema of $2 J_{1}(x) / x$ along the non-negative real axis coincides with the non-negative zeros of $J_{2}(x)$ (including $x_{0}=0$ as $2 J_{2}(x) / x \rightarrow 0$ when $x \rightarrow 0)$. Denote these zeros of $J_{2}(x)$ as $x_{0}=0<$ $x_{1}<x_{2}<\ldots<x_{k}<\ldots,(k \in N)$. According to recurrence relations, the local extrema of $2 J_{1}(x) / x$ takes values in $J_{0}\left(x_{k}\right)$ as $J_{2}\left(x_{k}\right)=0$. We now consider the integral representation of $J_{0}(x)$ : 


$$
J_{0}(x)=\frac{1}{\pi} \int_{0}^{\pi} \cos (x \sin \tau) \mathrm{d} \tau
$$

As the integral in (A4) is real-variant and realvalued, also noticing the fact that $|\cos x| \leqslant 1$ for any real $x$, we can see $\left|J_{0}(x)\right| \leqslant 1$. Therefore, the values at the local extrema of $2 J_{1}(x) / x$ fulfil $\left|2 J_{1}\left(x_{k}\right) / x_{k}\right|=\left|J_{0}\left(x_{k}\right)\right| \leqslant 1=\left|J_{0}\left(x_{0}\right)\right|$.

As the value of $J_{1}(x)$ is finite and $J_{1}(x) \rightarrow 0$ when $x \rightarrow \infty$, we know that $J_{0}(0)$ is not only a local maximum for $2 J_{1}(x) / x$, but also a global maximum for $\left|2 J_{1}(x) / x\right|$ as well, i.e.:

$$
\left|\frac{2 J_{1}(x)}{x}\right| \leqslant 1
$$

Combining the two terms, we reach the conclusion that $\left|\gamma_{0}\right| \leqslant 1$.

\section{APPENDIX B}

We prove (24) in a Cartesian coordinate system. Without loss of generality, let $\boldsymbol{s}=(0,0,1)$ and write $\boldsymbol{e}=(\cos \phi, \sin \phi, 0)$ and $\boldsymbol{h}=(-\sin \phi, \cos \phi, 0)$ for some $\phi \in[0,2 \pi)$. At a given $\varphi$, let $\boldsymbol{s}^{\prime}=$ $(\sin \varphi, 0, \cos \varphi)$, without loss of generality and for simplicity of calculations. Then, the plane expanded by $\boldsymbol{e}^{\prime}$ and $\boldsymbol{h}^{\prime}$ is given by plane equation $x \sin \varphi+$ $z \cos \varphi=0$.

Write $\boldsymbol{e}^{\prime}=(\sin \alpha \cos \varphi, \cos \alpha,-\sin \alpha \sin \varphi)$ where $\alpha \in[0,2 \pi)$, then it satisfies $\boldsymbol{e}^{\prime} \perp \boldsymbol{s}^{\prime}$ and $\left|\boldsymbol{e}^{\prime}\right|=1$. Hence, $\boldsymbol{h}^{\prime}$ is given by $\boldsymbol{h}^{\prime}=\boldsymbol{s}^{\prime} \times$ $\boldsymbol{e}^{\prime}=(-\cos \alpha \cos \varphi, \sin \alpha, \cos \alpha \sin \varphi)$. Then, we have $\boldsymbol{e} \cdot \boldsymbol{e}^{\prime}=\cos \phi \sin \alpha \cos \varphi+\sin \phi \cos \alpha$ and $\boldsymbol{h} \cdot \boldsymbol{h}^{\prime}=\sin \phi \cos \alpha \cos \varphi+\cos \phi \sin \alpha$.

It is now clear that $\boldsymbol{e} \cdot \boldsymbol{e}^{\prime}$ and $\boldsymbol{h} \cdot \boldsymbol{h}^{\prime}$ are only equal when $\varphi=0$ or $\sin (\phi-\alpha)=0$. So, $\mid \boldsymbol{e} \cdot \boldsymbol{e}^{\prime}+\boldsymbol{h}$. $\boldsymbol{h}^{\prime}|=(1+\cos \varphi)| \cos \phi \sin \alpha+\sin \phi \cos \alpha \mid=(1+$ $\cos \varphi)|\sin (\phi+\alpha)|$.

Since $|\sin (\phi+\alpha)| \leqslant 1$, it is now easy to spot that (24) holds true, i.e. $\left|\boldsymbol{e} \cdot \boldsymbol{e}^{\prime}+\boldsymbol{h} \cdot \boldsymbol{h}^{\prime}\right| \leqslant 1+\cos \varphi$. The ' $\leqslant$ ' becomes ' $=$ ' when $\sin (\phi+\alpha)= \pm 1$, or equivalently $\phi+\alpha=\pi / 2+k \pi(k=0,1,2,3)$.

\section{APPENDIX C}

In Appendix C, we explain the ICI-free property of the proposed CD SMX system.

The IM/DD SMX system would suffer from ICI because it uses direct detection, so the light/signal coming from all transmitters could possibly be received by a single receiver (as long as the signal falls within the field-of-view (FOV) of the said receiver).

The proposed CD SMX system is however free from the ICI because of the photomixing process. At a certain receiver, it is still possible that the signal coming from an unmatched transmitter falls within the FOV of this receiver, but this signal is not matched to the LO at this receiver. The results in (14) can be easily extended to the case where $n$ signals (including the LO light) are mixing: there would be $n$ terms corresponding to the selfmixing of the $n$ signals, and $n(n-1)$ cross terms corresponding to the mixing between any 2 different signals.

When $n$ signals are present, the Poynting vector of the total field would now be:

$$
\langle\mathbf{S}\rangle=\left(\sum_{k=1}^{n} \mathbf{E}_{k}\right) \times\left(\sum_{k=1}^{n} \mathbf{H}_{k}^{*}\right)
$$

where $\mathbf{E}_{k}$ and $\mathbf{H}_{k}$ are the electromagnetic fields of the $k$ th signal. We expand the summations in $(\mathrm{C} 1)$, and we can categorise the terms into two kinds: the self mixing terms $\mathbf{E}_{k} \times \mathbf{H}_{k}^{*}$, and the cross terms $\mathbf{E}_{i} \times$ $\mathbf{H}_{j}^{*}$ where $i \neq j$ :

$$
\langle\mathbf{S}\rangle=\sum_{k=1}^{n} \mathbf{E}_{k} \times \mathbf{H}_{k}^{*}+\sum_{1 \leqslant i \neq j \leqslant n} \mathbf{E}_{i} \times \mathbf{H}_{j}^{*}
$$

All the unmatched cross terms will be approximately zero for their poor photomixing efficiencies. So only the $n$ self-mixing terms and the 2 matched cross terms (corresponding to the matched signal and LO lasers) would remain non-zero, i.e. there would be $n$ DC terms and 1 cosine term in the photocurrent expression, excluding the noise terms. The photomixing of multiple simultaneous signals would only result in increased DC power, provided that the matching pattern is one-on-one. In other words, the proposed CD SMX system is ICI-free, assuming $\gamma_{i, j} \approx 0$ where $i \neq j$.

\section{ACKNOWLEDGEMENTS}

Professor Harald Haas greatly acknowledges support from the EPSRC under Established Career Fellowship Grant EP/R007101/1. He also acknowledges the financial support of his research by the Wolfson Foundation and the Royal Society. 
The authors would also like to thank their colleagues Dr. Iman Tavakkolnia, Dr. Elham Sarbazi and Ms. Hannah Brown for the useful comments and discussion.

\section{REFERENCES}

[1] H. Haas, L. Yin, Y. Wang, and C. Chen, "What is LiFi?" $J$. Lightw. Technol., vol. 34, no. 6, pp. 1533-1544, Mar. 2016.

[2] D. Tsonev, S. Videv, and H. Haas, "Towards a $100 \mathrm{~Gb} / \mathrm{s}$ visible light wireless access network," Opt. Express, vol. 23, no. 2, pp. 1627-1637, Jan. 2015.

[3] Y. Tan, X. Wu, and H. Haas, "Performance comparison between coherent and DCO-OFDM LiFi systems," in ICC 2019 - 2019 IEEE International Conference on Communications (ICC), May 2019, pp. 1-6.

[4] J. R. Barry, Wireless Infrared Communications. Boston, M.A.: Springer, 1994.

[5] J. R. Barry and E. A. Lee, "Performance of coherent optical receivers," Proc. IEEE, vol. 78, no. 8, pp. 1369-1394, Aug. 1990.

[6] M. S. Islim and H. Haas, "Modulation techniques for LiFi," ZTE communications, vol. 14, no. 2, pp. 29-40, 2016.

[7] T. Fath and H. Haas, "Performance comparison of MIMO techniques for optical wireless communications in indoor environments," IEEE Trans. Commun., vol. 61, no. 2, pp. 733-742, Feb. 2013.

[8] R. Mesleh, H. Haas, S. Sinanovic, C. W. Ahn, and S. Yun, "Spatial modulation," IEEE Trans. Veh. Technol., vol. 57, no. 4, p. $2228,2008$.

[9] H. G. Olanrewaju, J. Thompson, and W. O. Popoola, "Performance of optical spatial modulation in indoor multipath channel," IEEE Trans. Wireless Commun., vol. 17, no. 9, pp. 6042-6052, Sept. 2018.
[10] K. P. Peppas and P. T. Mathiopoulos, "Free-space optical communication with spatial modulation and coherent detection over H-K atmospheric turbulence channels," J. Lightw. Technol., vol. 33, no. 20, pp. 4221-4232, Oct. 2015.

[11] R. Mesleh, H. Elgala, and H. Haas, "Optical spatial modulation," IEEE/OSA J. Opt. Commun. Netw., vol. 3, no. 3, pp. 234-244, Mar. 2011.

[12] R. M. Gagliardi and S. Karp, Optical Communications. New York, Wiley-Interscience, 1976. 445 p., 1976.

[13] R. K. Tyson, Principles of Adaptive Optics, 3rd ed. Boca Raton: CRC Press, Sept. 2010.

[14] A. E. Siegman, "The antenna properties of optical heterodyne receivers," Appl. Opt., vol. 5, no. 10, pp. 1588-1594, Oct. 1966.

[15] D. J. Griffiths, Introduction to Electrodynamics, 3rd ed. Upper Saddler River, N.J. : London: Prentice Hall Prentice-Hall International, 1999.

[16] Status of IEEE 802.11 Light Communication TG. [Online]. Available: https://www.ieee802.org/11/Reports/tgbb_ update.htm

[17] Proposal for common mode PHY. [Online]. Available: $\quad$ https://mentor.ieee.org/802.11/dcn/19/ 11-19-1625-04-00bb-proposed-common-mode-mandatory-phy. pptx

[18] J. C. Dainty, Laser Speckle and Related Phenomena. Springer science \& business Media, 2013, vol. 9.

[19] I. Tavakkolnia, A. Yesilkaya, and H. Haas, "OFDM-based spatial modulation for optical wireless communications," in 2018 IEEE Globecom Workshops (GC Wkshps), Dec. 2018, pp. $1-6$.

[20] I. Stefan, H. Elgala, and H. Haas, "Study of dimming and LED nonlinearity for ACO-OFDM based VLC systems," in 2012 IEEE Wireless Communications and Networking Conference (WCNC), April 2012, pp. 990-994. 